\title{
A self-consistent theoretical framework for magnetized accretion discs
}

\author{
Geoffrey V. Bicknell ${ }^{1}$ \\ and Zdenka Kuncic ${ }^{2}$ \\ ${ }^{1}$ Mt. Stromlo Observatory, Research School of Astronomy \& Astrophysics, Cotter Rd., \\ Weston, ACT 2611, Australia email: geoff@mso.anu.edu.au \\ ${ }^{2}$ School of Physics, University, Sydney, NSW 2006, Australia \\ email: kuncic@physics.usyd.edu.au
}

\begin{abstract}
Magnetic fields are crucial to the generation of turbulence and the dynamics of accretion and energy dissipation in accretion disks. Nevertheless, until now, there has been no self-consistent analytic formalism that puts the evolution of turbulent magnetic fields on the same footing as other dynamical processes such as mass flow and energy generation. We have developed a self-consistent framework for turbulent, magnetized accretion disks with the aim of studying the processes that power hot coronae. Utilizing this framework we have identified the possible magnetic sources of power for the coronae of black hole accretion disks. The most promising is the Poynting flux associated with the azimuthal-vertical magnetic stress. We also determine how the emission from the disk is modified by the processes that power the corona and related winds.
\end{abstract}

\section{Introduction}

The notion that accretion discs become turbulent because of the presence of weak magnetic fields is now well known through the work of Balbus, Hawley, Stone and collaborators (e.g. Balbus \& Hawley 1998; Miller \& Stone 2000). The purpose of this work, a complete desciption of which is presented in Kuncic \& Bicknell (2004), is to develop an analytical framework for accretion discs that incorporates the effects of turbulent magnetic fields $a b$ initio and which can be used as the basis for further analytical and numerical investigations. One of the motivations driving this work has been the existence of accretion disc coronae and the issues of how energy generated in the accretion disk is propagated into the corona and dissipated there and how this relates to disk outflows. Blandford \& Payne (1982) and Pudritz \& Norman (1983) have demonstrated the existence of outflows in the case of a strong magnetic field when nett nagentic flux threads the disc. The initial focus of our work is to examine the issue of outflows in the case of zero nett magnetic flux.

\section{The Poynting flux}

Haardt \& Maraschi (1991) developed a phenomenological model for accretion disk coronae that requires a substantial flux ( $\sim$ the accretion power $)$ of energy into the corona above the disk. An obvious candidate is the Poynting flux, expressed in cylindrical $r(, \phi, z)$ coordinates as

$$
S_{z} \approx \frac{B_{r}^{2}+B_{\phi}^{2}}{4 \pi} v_{z}-\frac{B_{\phi} B_{z}}{4 \pi} v_{\phi}
$$

where $v_{z}$ is the component of velocity perpendicular to the disk and $v_{\phi}$ is the azimuthal Keplerian velocity $\simeq(G M / r)^{1 / 2}$. Some key questions posed by this expression are (1) 
Does a vertical wind or buoyant velocity provide a sufficient Poynting flux? and (2) What is the magnitude of the Poynting flux associated with the Keplerian velocity (the second term in equation $(2.1))$ ?

\section{Equations for the mean flow}

\subsection{Statistically averaged equations}

In order to develop an analytic model for turbulent, magnetized accretion disks, we find it useful to utilize the statistical mass-averaging technique introduced by Favre (1969). In this method, all variables are expressed in terms of mean and fluctuating components, with extensive variables such as the density averaged in a conventional way but intensive variables such as velocity are mass-averaged. Thus, for example, denoted ensemble (or time averages) by \langle\rangle

$$
\begin{array}{rrrrrr}
\text { Density: } & \rho=\bar{\rho}+\rho^{\prime} & \left\langle\rho^{\prime}\right\rangle=0 & \text { Velocity: } & v_{i}=\tilde{v}_{i}+v_{i}^{\prime} & \left\langle\rho v_{i}^{\prime}\right\rangle=0 \\
\text { Pressure: } & p=\bar{p}+p^{\prime} & \left\langle p^{\prime}\right\rangle=0 & \text { Magnetic Field: } & B_{i}=\bar{B}_{i}+B_{i}^{\prime} & \left\langle B_{i}^{\prime}\right\rangle=0
\end{array}
$$

In this treatment we assume zero nett flux through the disk (i.e. $\left\langle\bar{B}_{i}\right\rangle=0$ ). Taking the ensemble average of the MHD equations introduces several additional terms into the equations for the mean flow. For example, the momentum equations become:

$$
\frac{\partial\left(\bar{\rho} \tilde{v}_{i}\right)}{\partial t}+\frac{\partial\left(\bar{\rho} \tilde{v}_{i} \tilde{v}_{j}\right)}{\partial x_{j}}=-\bar{\rho} \frac{\partial \phi_{\mathrm{G}}}{\partial x_{i}}-\frac{\partial \bar{p}}{\partial x_{i}}+\frac{\partial}{\partial x_{j}} \frac{\partial\left\langle t_{i j}\right\rangle}{\partial x_{j}}
$$

where $\phi_{\mathrm{G}}$ is the gravitational potential, $p$ is the thermal plus radiation pressure and the total turbulent stress is the sum of the Reynolds and magnetic stresses, i.e.

$$
\left\langle t_{i j}\right\rangle=-\left\langle\rho v_{i}^{\prime} v_{j}^{\prime}\right\rangle+\frac{\left\langle B_{i}^{\prime} B_{j}^{\prime}\right\rangle}{4 \pi}-\frac{\left\langle B^{\prime 2}\right\rangle}{8 \pi} \delta_{i j}
$$

\subsection{Thin disk approximation}

$\mathrm{Al}$ of the dynamical equations can be put into a conservation law form and integrated over the height of the disk, whose $\tau=1$ surfaces $(\tau=$ optical depth) are located at $z= \pm h$. Thus the generic conservation law:

$$
\frac{1}{r} \frac{\partial\left(r A_{r}\right)}{\partial r}+\frac{\partial A_{z}}{\partial z}=S \Rightarrow \frac{\mathrm{d}}{\mathrm{d} r} \int_{-h}^{+h} 2 \pi r A_{r} \mathrm{~d} z+2 \pi r\left(A_{z}^{+}-A_{z}^{-}\right)=\int_{-h}^{+h} 2 \pi r S \mathrm{~d} z
$$

with the photosphere-corona boundary denoted by the \pm superscript.

\subsubsection{Mass flux}

There are two important mass fluxes:

$$
\begin{aligned}
\text { Mass accretion rate: } & \dot{M}_{\mathrm{a}}(r)=-2 \pi \int_{-h}^{+h} r \bar{\rho} \tilde{v}_{r} d z \\
\text { Wind mass loss rate: } & \dot{M}_{\mathrm{w}}(r)=4 \pi \int_{r}^{\infty} r \bar{\rho}^{+} \tilde{v}_{z}^{+} d r
\end{aligned}
$$

with mass conservation telling us that $\dot{M}_{\mathrm{a}}(r)+\dot{M}_{\mathrm{w}}(r)=\dot{M}=$ accretion rate at $r=\infty$.

\subsubsection{Radial and vertical momentum balance}

The radial and vertical momentum balances are identical to the standard Shakura \& Sunyaev (1976) disk. We define the disk surface density, $\Sigma$ and average scale height $h_{\mathrm{av}}$ by:

$$
\Sigma(r)=\int_{-h}^{+h} \bar{\rho} d z \quad h_{\mathrm{av}}(r)=\frac{\int_{-h}^{+h} \bar{\rho} z d z}{\Sigma(r)}
$$


When the stress $\left\langle t_{r z}\right\rangle<\bar{\rho} G M / r$ the azimuthal velocity is Keplerian, i.e. $\tilde{v}_{\phi} \simeq(G M / r)^{1 / 2}$. When the vertical velocity in the disk is less than the sound speed then the disk is in a quasiequilibrium state and $h_{\mathrm{av}} / r \sim c_{0}^{\text {tot }} / v_{\mathrm{K}}$, where mid-plane sound speed, $c_{0}^{\text {tot }}=$ $\left(\left(\bar{p}_{0}+\left\langle B_{0}^{2}\right\rangle / 8 \pi\right) / \rho_{0}\right)^{1 / 2}$ includes the radiation pressure and magnetic field.

\subsection{Angular momentum}

Solution of the angular momentum equation gives for the integrated $r \phi$ stress, $T_{r \phi}=$ $\int_{-h}^{+h} t_{r \phi} d z$ :

$T_{r \phi}(r)=-\frac{\dot{M}_{\mathrm{a}}(r) v_{\mathrm{K}}(r)}{2 \pi r}\left[1-\frac{\dot{M}_{\mathrm{a}}\left(r_{i}\right) r_{i} v_{\mathrm{K}}\left(r_{i}\right)}{\dot{M}_{\mathrm{a}}(r) r v_{\mathrm{K}}(r)}\right]+\frac{r_{i}^{2} T_{r \phi}\left(r_{i}\right)}{r^{2}}+\int_{r_{i}}^{r} \frac{r^{\prime 2}}{r^{2}}\left[\bar{\rho}^{+} v_{\mathrm{K}} \tilde{v}_{z}^{+}-\left\langle t_{\phi z}^{+}\right\rangle\right] d r^{\prime}$

The first term on the right represents the standard result (apart from the effect of a varying accretion rate); the other terms represent the effects of the inner disk stress, the wind and the $\phi z$ stress. The $r \phi$ and $\phi z$ stress terms in this equation are comparable when $\left\langle t_{\phi z}\right\rangle^{+} \sim h_{\mathrm{av}} / r\left\langle t_{r \phi}\right\rangle \sim 10^{-3}\left\langle t_{r \phi}\right\rangle_{0}$, reflecting the fact that the $\phi z$ stress acts over the surface of the disk but the $r \phi$ term acts across the disk cross-section. Moreover, the $\phi z$ stress (dominated by the magnetic field) has an important effect on the transport of angular momentum when

$$
\frac{-4 \pi r^{2}\left\langle t_{\phi z}\right\rangle^{+}}{\dot{M}_{\mathrm{a}} v_{\mathrm{K}}} \sim \frac{-4 \pi r^{2}\left\langle B_{\phi}^{\prime} B_{z}^{\prime}\right\rangle^{+}}{\dot{M}_{\mathrm{a}} v_{\mathrm{K}}} \sim 1
$$

leading to the numerical estimate for the magnetic field:

$\left\langle-B_{\phi}^{\prime} B_{z}^{\prime}\right\rangle^{1 / 2} \approx\left(\frac{m_{p} c^{4}}{G \sigma_{T}}\right)^{1 / 2}\left(\frac{\dot{m}}{M}\right)^{1 / 2}\left(\frac{r}{r_{g}}\right)^{-5 / 4}=7 \times 10^{2}\left(\frac{\dot{m}}{M_{8}}\right)^{1 / 2}\left(\frac{r}{10 r_{g}}\right)^{-5 / 4}$ Gauss

where $\dot{m}$ is the mass accretion rate in units of the Eddington value, the mass of the black hole is $10^{8} M_{8} M_{\odot}$ and $r_{g}$ is the gravitational radius.

\section{Disk luminosity}

Solution of the total energy equation for the disk gives an equation for the radiative flux through the disk surface, integration of which gives the following equation for the disk luminosity:

$$
L_{\text {disk }}=\frac{G M \dot{M}_{\mathrm{a}}\left(r_{i}\right)}{2 r_{i}}-2 \pi r_{i} v_{\mathrm{K}}\left(r_{i}\right) T_{r \phi}\left(r_{i}\right)-P_{\text {wind }}
$$

The first two terms represent the usual gravitational power and the effect of a nonvanishing stress at the inner disk boundary, respectively. The power in the wind is dominated by kinetic, gravitational, magnetic terms and is given by:

$$
P_{\text {wind }}=\int_{r_{i}}^{\infty}\left[\frac{-G M}{2 r} \bar{\rho}^{+} \tilde{v}_{z}^{+}+\frac{\left\langle B_{r}^{\prime 2}+B_{\phi}^{\prime 2}\right\rangle^{+}}{4 \pi} \tilde{v}_{z}^{+}+\frac{\left\langle\left(B_{r}^{\prime 2}+B_{\phi}^{\prime 2}\right) v_{z}^{\prime}\right\rangle}{4 \pi}-\frac{\left\langle B_{\phi}^{\prime} B_{z}^{\prime}\right\rangle^{+}}{4 \pi} v_{\mathrm{K}}\right] 4 \pi r d r
$$

The terms in $P_{\text {wind }}$ include the (negative) flux of kinetic plus gravitational potential energy and three Poynting flux terms related to advection and buoyant turbulent diffusion of magnetic energy plus a term proportional to the product of the $\phi z$ magnetic stress and the Keplerian velocity. Detailed comparison of the various terms shows that it is most 
likely the last term that dominates since it involves the Keplerian velocity. Thus, we have the very interesting situation that the stress component $\left\langle t_{\phi z}\right\rangle$ can have a dominant effect on the energy transfer in an accretion disk-corona system even though it is numerically small. The last term also figures in the condition for a wind. The energy flux density in the wind is positive (i.e. the last Poynting flux component counteracts the negative kinetic and potential energy component) when

$$
\frac{-\left\langle B_{\phi}^{\prime} B_{z}^{\prime}\right\rangle^{+}}{\left\langle B^{\prime 2}\right\rangle} \frac{\left\langle v_{\mathrm{A}}^{2}\right\rangle^{+}}{v_{\mathrm{K}}^{2}} \gtrsim \frac{h_{\mathrm{av}}}{r} \frac{\tilde{v}_{z}^{+}}{c_{0}}
$$

This condition can be readily satisfied when $\left\langle v_{\mathrm{A}}^{2}\right\rangle^{+}>v_{\mathrm{K}}^{2}$ but could also be satisfied for much lower magnetic fields, reminding one of the "magnetic switch" from loosely collimated wind flow to jet flow (Meier 1999).

\section{Accretion power and wind power}

Disks in which the dominant stress is $\left\langle t_{r \phi}\right\rangle$ and $\left\langle t_{\phi z}\right\rangle=0$ have dominated the field ever since the work of Shakura and Sunyaev. Ignoring the effect of stress on the inner boundary, such a disk is characterized by:

$$
L_{\text {disk }}=\frac{G M \dot{M}_{\mathrm{a}}\left(r_{i}\right)}{2 r_{i}} \quad P_{\text {wind }}=0
$$

The other extreme is one in which $\left\langle t_{r \phi}\right\rangle=0$ and $\left\langle t_{\phi z}\right\rangle \neq 0$ which gives:

$$
L_{\text {disk }}=0 \quad P_{\text {wind }}=\frac{G M \dot{M}_{\mathrm{a}}\left(r_{i}\right)}{2 r_{i}}
$$

i.e. all of the gravitational power ends up in the wind. Real disks are probably intermediate between the two extremes. However, this is the first time that the partition of power between disk luminosity and winds has been put on a physical basis.

\section{Concluding remarks}

We have established a self-consistent framework for magnetized accretion disks and have shown that the $\phi z$ magnetic stress can have an important dynamical and energetic effect even though it may be numerically small compared to to other stresses. Significant gravitational accretion power can be channeled into the corona via the Poynting flux associated with this stress. Future work will involve the nature of wind flows from radiative magnetized disks and the manner in which Poynting flux can be dissipated in the corona.

\section{References}

Balbus, S. A., \& Hawley, J. F. 1998, Rev. Mod. Phys., 70, 1

Blandford, R. D., \& Payne, D. G. 1982, MNRAS, 199, 883

Favre, A. 1969, in Statistical Equations of Turbulent gases, 231, (Philadelphia, Pennsylvania: Society for Industrial and Applied Mathematics)

Haardt, F., \& Maraschi, L. 1991, ApJ, 380, L51

Kuncic, Z., \& Bicknell, G. V. 2004, ApJ, submitted, astro-ph/0402421

Meier, D. L. 1999, ApJ, 522, 753

Miller, K. A., \& Stone, J. M. 2000, ApJ, 534, 398

Pudritz, R. E., \& Norman, C. A. 1983, ApJ, 274, 677

Shakura, N. I., \& Sunyaev, R. A. 1976, MNRAS, 175, 613 